\title{
Pathological implication and function of Bcl2-inhibitor of transcription in ovarian serous papillary adenocarcinomas
}

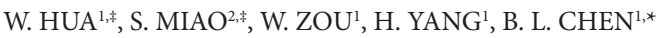 \\ ${ }^{1}$ Department of Obstetrics and Gynecology, Xijing Hospital, The Fourth Military Medical University, Xian, P.R. China; ${ }^{2}$ Institute of Materia \\ Medica, School of Pharmacy, The Fourth Military Medical University, Xian, P.R. China \\ ${ }^{*}$ Correspondence: blchenxian@gmail.com \\ ${ }^{*}$ Contributed equally to this work.
}

Received April 28, 2012/ Accepted September 12, 2012

\begin{abstract}
The Bit-1 protein appears to be a part of the integrin-specific signaling pathway involved into anoikis. When Bit1 is released from the mitochondria into the cytoplasm it can elicit caspase-independent apoptosis. The expression of Bit1 in 78 serous papillary adenocarcinomas and 78 normal epithelial ovarian tissue specimens was analyzed by immunohistochemistry. We also investigate Bit1 function by transfection. Bit1 was expressed in 100\% and 33.3\% of ovarian cancers and normal epithelial tissues, respectively, and its expression was significantly correlated with histologic grade and overall survival. However, Bitl expression was not associated with age. We also confirmed that Bit1 overexpression in cytosol of Caov- 3 cells induced apoptosis. Bit1 may be a useful pathological marker and a prognostic marker for serous papillary adenocarcinomas outcome. Its pro-apoptotic property also makes it a potential gene medicine for ovarian cancers therapy.
\end{abstract}

Key words: Bit1 (Bcl2-inhibitor of transcription), serous papillary adenocarcinomas, Apoptosis, Anoikis

Ovarian cancer is one of the most lethal gynaecological cancers worldwide. Approximately 1 in 75 women are diagnosed with this disease in the developed world, and over $75 \%$ of women present at an advanced stage, with disease spreading beyond the ovaries. Despite high rates of response to initial chemotherapy, the majority of women relapse, eventually with drug-resistant disease. The overall 5-year survival rate for women with advanced disease is only $30 \%[1,2]$.

Tumor cells are more resistant to anoikis (the subset of apoptosis triggered by inadequate or inappropriate cell-matrix contacts) than normal cells. This may provide a survival advantage to malignant cells during invasion and metastasis because tumor cells may bypass integrin-dependent survival signals [3]. Although various growth factors activate some of the same signaling pathways, they cannot replace integrinmediated attachment [4]. Hence, integrin-specific signaling pathways play a critical role in cell survival and the regulation of apoptosis.

Bit-1(Bcl2-inhibitor of transcription 1) [5] is identified as a part of the integrin-specific signaling pathway [4]. Unlike bacterial Bit1, the 22-kDa Bit1 polypeptide of archaea and eukaryotes uniquely contains an $\mathrm{N}$-terminal mitochondrial localization domain that is 179 amino acids long[4]. Bit1 can serve as a pro-apoptotic factor, playing a critical role in caspase-independent apoptosis known as anoikis. Upon loss of cellular attachment of both normal cells and tumor cells, Bit1 is released from the mitochondria into the cytoplasm and elicits apoptosis. Suppression of Bit1 expression significantly protects cells from anoikis. Moreover, when Bit-1 is experimentally targeted to the cytoplasm (by mutation) in attached cells, it binds to AES, a small Groucho/TLE (transducin-like enhancer of split) protein [4]. This AES-Bit1complex promotes anoikis[6]. Cell attachment mediated by integrins controls Bit1-induced apoptosis, whereas various other anti-apoptotic signaling molecules, such as phosphatidylinositol 3-kinase, and Akt, $\mathrm{Bcl}-2, \mathrm{Bcl}-\mathrm{xL}$, have no effects on Bit1-conducted apoptosis. Uncontrolled cell apoptosis is one of the main mechanisms of tumorigenesis, so studies on apoptosis related proteins such as Bit1 will be of great importance to the determination of tumorigenesis and tumor development.

However, to our knowledge, Bit1 distribution in relation to ovarian cancers has not been studied. To evaluate the potential clinical value of Bit 1 in ovarian cancers, we analyzed the expression of Bit1 in 78 serous papillary adenocarcinomas. 
We also transfected Bit1 cDNA into ovarian cancer cells to further investigate the potential role of Bit1. Our findings shed light on the potential use of Bitl as a biomarker for ovarian cancer diagnosis or as a target for ovarian cancer therapy.

\section{Materials and methods}

Reagents. Polyclonal antibodies against Bit 1 were a generous gift from Dr. Yuanming Wu, of The Fourth Military Medical University. A Streptavidin-peroxidase (SP) kit was purchased from Zhongshan corporation(Beijing,China). pMD-18T plasmids, Taq polymerase, DNA ladder kit, a PCR core kit, endonucleases EcoRI and SalI were all obtained from TaKaRa(Dalian, China). Lipofectamine 2000, T4 DNA ligase, Dulbecco's modified eagle medium (DMEM) and fetal bovine serum (FBS) were purchased from Invitrogen (Carlsbad, NM). An RNeasy kit and RT-PCR kit were purchased from Qiagen (Valencia, CA.). HPR-conjugated goat antimouse IgG was obtained from Pierce (Rockford, Illinois). Human ovarian papillary adenocarcinoma cell line (Caov-3), human umbilical vein endothelial cells (HUVECs) and Hela cells were obtained from American Tissue and Cell Culture (ATTC; Manassas, VA). The expression vector pCMV-Myc was obtained from BD Corporation (Franklin Lakes, NJ). An ECL detection system was purchased from Amersham (Arlington Heights, IL). MTT, DMSO and ethidium bromide were obtained from Sigma-Aldrich (Saint Louis, MO.).

Patients and tissue samples. Tissue samples from 78 serous papillary adenocarcinomas tissues diagnosed between 1999 and 2004 at Xijing hospital, China, were included in this study. The cancers were staging according to the International Federation of Gynecology and Obstetrics (FIGO) staging system 2000 [7]. The clinicopathological features of the 78 serous papillary adenocarcinomas tissues were shown in Table1. No patients had any treatment before the biopsy. This study was approved by the ethics committee of Shannxi Province Medical Association, China. All patients gave informed consent according to institutional guidelines and remained under continuous medical supervision and assistance in accordance with the Declaration of Helsinki.

Table I. The Bit1 expression in ovarian cancer tissues with different clinicopathologic factors

\begin{tabular}{cccccc}
\hline parameter & $\begin{array}{c}\text { No. } \\
\text { of } \\
\text { cases }\end{array}$ & Negative & $\begin{array}{c}\text { Bit } 1 \\
\text { Weak } \\
\text { positive }\end{array}$ & Positive & P value \\
\hline $\begin{array}{c}\text { Histologic } \\
\text { G1 }\end{array}$ & 32 & 0 & 15 & 17 & $\mathrm{P}=0.003,0.042<0.05$ \\
G2 & 33 & 0 & 23 & 9 & \\
G3 & 13 & 0 & 13 & 1 & NS \\
Age & & & & & \\
$<50$ & 39 & & 12 & 27 & \\
$\geq 50$ & 43 & & 11 & 31 & \\
\hline
\end{tabular}

For the analysis of survival and follow-up, the date of surgery was used to represent the beginning of the follow-up period. All the patients who died from diseases other than ovarian cancer or from unexpected events were excluded from the analysis. Follow-up ceased on November 10, 2011. Treatment modalities after relapse were applied according to a standard guideline.

Immunohistochemistry. Polyclonal antibodies against Bit1were used at a dilution of 1:100 in this assay. Human Ab serum was used as a negative control. The assay was performed as described previously [8]. In brief, five-micrometer sections of the paraffin-embedded, formalin-fixed tissue blocks were placed in $3 \% \mathrm{H}_{2} \mathrm{O}_{2}$ to block endogenous peroxidase at RT for $10 \mathrm{~min}$, and washed three times with PBS. The sections were then pretreated for antigen retrieval by microwaving in $10 \mathrm{mM}$ citrate buffer ( $\mathrm{pH}$ 6.0) for 15 minutes at high power. The sections were then incubated with the primary antibody overnight at $4{ }^{\circ} \mathrm{C}$ and washed with PBS containing $0.1 \%$ Tween three times (5 min each). After washing, the slides were incubated with biotin-labeled secondary antibody for $10 \mathrm{~min}$ at RT and washed again. Staining was visualized using avidinbiotin complex and counterstaining was performed with hematoxylin.

Scoring. The immunoreactive sections were evaluated independently by two individuals who were unaware of the clinicopathological factors and the patients' clinical outcome. At least 20 high-power fields were randomly chosen, and 2000 cells were counted for each case. The positive signal of Bit1 is brown, and is located in cytosol. Scoring proceeded as previously described [9]. Scoring for Bit1 included additional intervals of $\leq 10 \%,>10 \%$ and $\leq 50 \%$, and $>50 \%$. Specimens with less than $10 \%$ positive cells were considered Bit 1 negative; $>10 \%$ and $\leq 50 \%$, weak positive; $>50 \%$, positive. Reproducibility of scoring was $>85 \%$ both within and between scorers.

Cell culture. Human ovarian papillary adenocarcinoma cell line (Caov-3) cells, Hela cells and human umbilical vein endothelial cells (HUVECs) were cultured in Dulbecco's modified Eagle's medium (DMEM) with 10\% fetal bovine serum (FBS) and incubated at $37^{\circ} \mathrm{C}$ under standard conditions of $100 \%$ humidity, $95 \%$ air and $5 \% \mathrm{CO}_{2}$.

pCMV-Myc-Bit 1 constructs and transfection. Full-length Bit1 cDNA was cloned from Hela cells (sense:5'-CGAATTCCCATGGTGAGCAAGGGCGAG-3', ant is ence: 5' - GGCTCGAGTCATTACTTGTACAGCTCGTCCAT $\left.-3^{\prime}\right)$ as previously described[10] and then cloned into pUC-18. pCMV-Myc-Bit1 recombinants were constructed from pCMV-Myc and pUC18-Bit1 and were transfected into Caov-3 cells using Lipofectamine 2000 reagent according to the manufacture's protocol. Resting cells were employed as the negative control. After the indicated time of transfection, cells were collected and used for subsequent investigations.

Cell viability assay. Cell viability was assessed using the MTT method at the indicated time after transfection [11]. Briefly, Cells were pulsed with $20 \mu \mathrm{l}$ of a $5 \mathrm{mg} / \mathrm{ml} \mathrm{MTT} \mathrm{stock}$ 


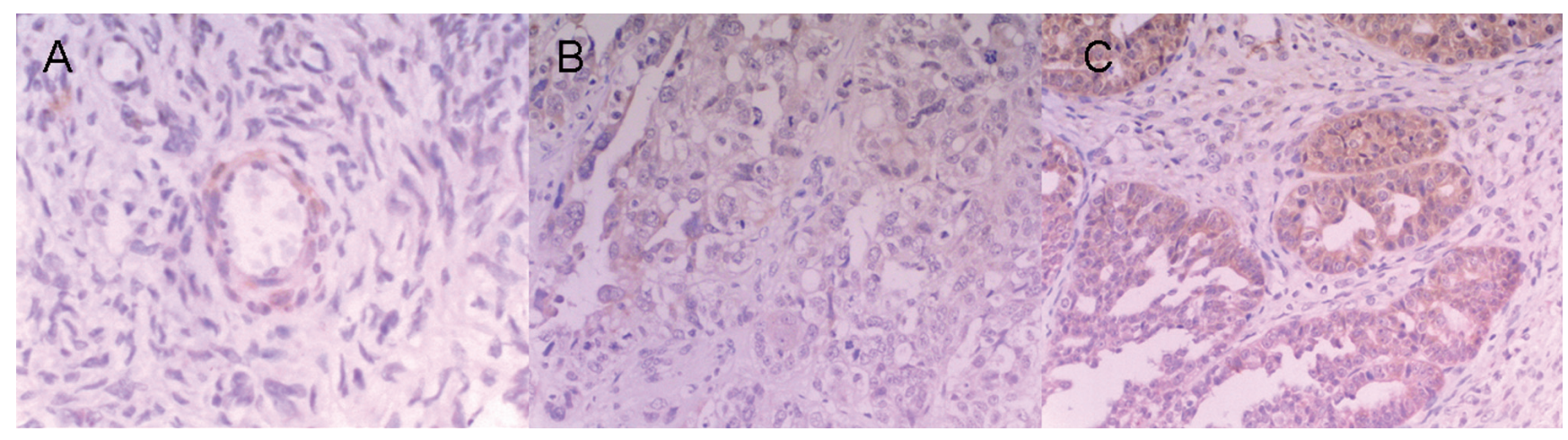

Figure1. Immunohistochemical analysis of Bit1 expression in normal epithelial ovarian tissues and serous papillary adenocarcinoma. The staining of Bit1 protein was mainly located in cytoplasm. (A) Normal epithelial ovarian tissue, weak positive Bit1 expression; (B) serous papillary adenocarcinomas, weak positive Bit1 expression; (C) serous papillary adenocarcinomas, positive Bit1 expression

in PBS and incubated for $4 \mathrm{~h}$, after which $150 \mu \mathrm{l}$ of DMSO was added. The plates were then plated on a shaker for 10min, and absorption was read on a VICTOR 3 multi-label plate reader (PerkinElmer) using a test wavelength of $490 \mathrm{~nm}$. Test reagents were added to medium alone to provide a blank.

Acridine orange staining. he apoptosis of Caov-3 cells were assessed by staining with acridine orange. Cells were harvested in trypsin at a certain time and were suspended in PBS. $5 \mu \mathrm{L}$ of acridine orange solution $(0.1 \mathrm{mg} / \mathrm{ml}$, diluted with PBS) was added to $95 \mu \mathrm{L}$ of cell suspension and the mixture was observed under a fluorescence microscope (Olympus).

DNA fragmentation assay. Transfected cells were harvested at the indicated time and were adjusted to $2 \times 10^{6} / 200 \mu \mathrm{l}$ with PBS. DNA fragments were purified according to the DNA ladder kit, and dissolved in $50 \mu \mathrm{L}$ of TE. Purified DNA $(5 \mu \mathrm{L})$ from each sample was loaded onto a $1 \%$ agarose gel, electrophoresed for $30 \mathrm{~min}$ at 75 volts and visualized under ultraviolet light after ethidium bromide staining.

Flow cytometry assay. Transfected cells were harvested in trypsin and washed three times with PBS at the indicated time as previously described [12]. Then cells were suspended in PBS and adjusted to $1 \times 10^{6} \mathrm{cells} / \mathrm{ml}$. $2 \mathrm{~mL}$ of cold ethyl alcohol $\left(4^{\circ} \mathrm{C}\right)$ was added to cells and the mixture was fixed at $4^{\circ} \mathrm{C}$ for $24 \mathrm{~h}$. Prior to addition of PI, cells were centrifuged and washed with PBS. Variation of DNA content was detected by flow cytometry (BD Corporation) [13].

RNA isolation and RT-PCR. Total cellular RNA was isolated using Trizol reagent according to the manufacture's instruction. First strand cDNA was prepared from $1 \mu \mathrm{g}$ of total cellular RNA in $20 \mu \mathrm{L}$ of reaction volume using 20 $\mathrm{U}$ of $\mathrm{M}-\mathrm{MuLV}$ reverse transcriptase. Primes were as follows: Bit1: sense, 5'-GGAACTTGTCACCCAGGAAC-3', antisense, 5'CTTGTGATGGAGGGGTTGTT-3'; $\beta$-actin sense: 5'AGAAGGATTCCTATGTGGGGG-3', Anti-sense:5'-CATGTCGTCCCAGTTGGTGAC-3'. Amplification was conducted using the PCR core kit. Five to $10 \mu \mathrm{L}$ of the reaction mixture was separated on a $1.5 \%$ agarose gel, and specifically amplified products were detected by ethidium bromide staining and UV transillumination. Semiquantitative analysis was conducted using a computerized densitometric image (Edison).

Western blotting. Caov-3 cells were homogenized using lysis buffer (PBS, $1 \%$ nonidet P-40 (NP-40), $0.5 \%$ sodium deoxycholate, $0.1 \%$ sodium dodecyl sulfate (SDS), $100 \mathrm{ug} / \mathrm{ml}$ aprotinin, $100 \mu \mathrm{g} / \mathrm{ml}$ phenylmethylsulfonyl fluoride (PMSF), Sodium orthovanadate) for $40 \mathrm{~min}$ at $4^{\circ} \mathrm{C}$. The protein content was determined according to Bradford's method (Bradford 1976), with bovine serum albumin used as a standard. For western blotting, equal amounts of protein were boiled with $2 \times$ sample buffer containing $5 \% \beta$-mercaptoethanol for $5 \mathrm{~min}$ and resolved on tris-glycine gels and electrophoretically transferred to nitrocellulose membrane at $90 \mathrm{~V}$ for $2 \mathrm{~h}$. The membranes were incubated with primary antibodies at $4^{\circ} \mathrm{C}$ overnight followed by HRP-conjugated secondary antibodies. Membranes were developed using the ECL detection system.

Statistical analysis. All assays were performed at least twice with duplicate or triplicate samples in each experiment. Statistical analysis was performed using a paired Student's t test. A $p$ value of $<0.05$ was considered significant.

\section{Results}

Association of Bit1 expression and clinicopathological parameters. The clinical characteristics of the study cohort are shown in Table I. Bit1 1 expression was immunohistologically studied in a total of 78 serous papillary adenocarcinomas. Seventy-eight specimens of normal epithelial ovarian tissues served as the control group (Figure1). Bit1 were expressed in $100 \%$ of 78 serous papillary adenocarcinomas tissues but weak expressed in only $33.3 \%$ of the 78 normal epithelial ovarian tissues, with a significant difference between these two types of tissues $(P<0.05)$ (Table I). Strong positive expression of Bit1 was observed in $53.1 \%, 28.1 \%$ and $7.1 \%$ of different grades of serous papillary adenocarcinoma, respectively, with a significant difference between G1 grade and G2 grade or G3 grade 


\section{Survival proportions}

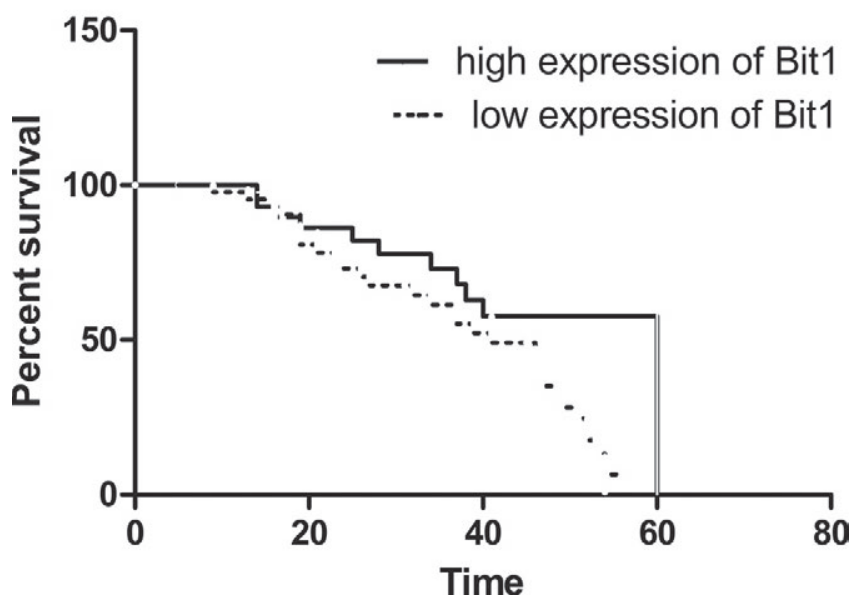

Figure 2. Statistical analysis of Bit1 protein levels and overall survival using the Kaplan-Meier method. There was a significant different overall survival of patients with G1 grade serous papillary adenocarcinoma and patients with G2/G3 grade serous papillary adenocarcinoma $(\mathrm{P}<0.05)$.

ovarian cancer tissues $(P=0.042,0.003$, both $<0.05)$. There was no significant difference in the expressions of Bit1 between G2 grade and $\mathrm{G} 3$ grade ovarian cancer tissues $(P=0.143>0.05)$. We also analyzed the association of Bit1 and age, and found no significant difference between age groups. Thus Bit1 expressions in serous papillary adenocarcinoma was associated with histologic grade but not with age.

Survival analyses. A high histologic grade was associated with 5-year overall survival in a univariate analysis. The results of an analysis of overall survival using the Kaplan-Meier method are shown in Figure 2. During the follow-up period, the 5-year overall survival rates for G1 grade and G2/G3 grade ovarian cancer patients were $65 \%$ and $34 \%$, respectively, and the overall survival rates showed significant difference between $\mathrm{G} 1$ grade and $\mathrm{G} 2 / \mathrm{G} 3$ grade $(\mathrm{P}<0.05)$. These results suggested that low expression of Bit1 in high histologic grade of serous papillary adenocarcinoma was associated with low overall survival of the patients.

Over expression of Bit1 in ovarian cancer cells has no influence on cell cycle but decrease cell viability. To further elucidate the function of Bit1 in ovarian cancer, we successfully constructed pCMV-Myc-Bit1 recombinant vectors and transfected them into ovarian cancer cell line Caov-3 cells. The expression of Bit1 constructs in cytosol was assessed by western blotting and RT-PCR. As shown in Figure 3A, Caov3 cells without transfection or transfected with pCMV-Myc and normal human cell HUVECs showed a low level of Bit1 mRNA. In Caov-3 cells transfected with pCMV-Myc-Bit1 recombinants, Bit1mRNA levels was significantly enhanced $(\mathrm{P}<0.05)$. Western blotting analysis showed that Bit1 protein levels were significantly enhanced in pCMV-Myc-Bit1-transfected Caov-3 cells $(\mathrm{P}<0.05$, Fig 3B).

DNA content of Bit 1 overexpressing cells was measured by flow cytometry. Cells growing exponentially were most in G1 phase followed by $S$ phase, and then $\mathrm{G} 2 / \mathrm{M}$. The proportion of cells in different phases did not appear to differ with transfection or different culture times (Figure $4 \mathrm{~A}-\mathrm{C}$ ). These results suggested that overexpression of Bit1 in Caov-3 cytosol did not affect the cell cycles.

To assess the viabilities of Bit1-overexpressing cells, we performed MTT. The results are shown in Figure 5. The viabilities of HUVECs and Caov-3 cells without transfection or transfected with pCMV-Myc appeared to be unaffected, and cell number increased in a time-depend fashion. On the contrary, the viability of the experimental group decreased as culture progressed, and cell growth was slow. Significant differences were observed between pCMV-Myc-Bit1 transfection group and the other groups. These results suggested that overexpression of cytosolic Bit1 had an inhibitory effect on cell viability.

Over expression of Bit1 in ovarian cancer cells promote apoptosis. Bit1 locating in the mitochondria does not bind to the cytoplasmic AES complex or promote cell death. However, when released from mitochondria into cytosol, Bit1 promotes

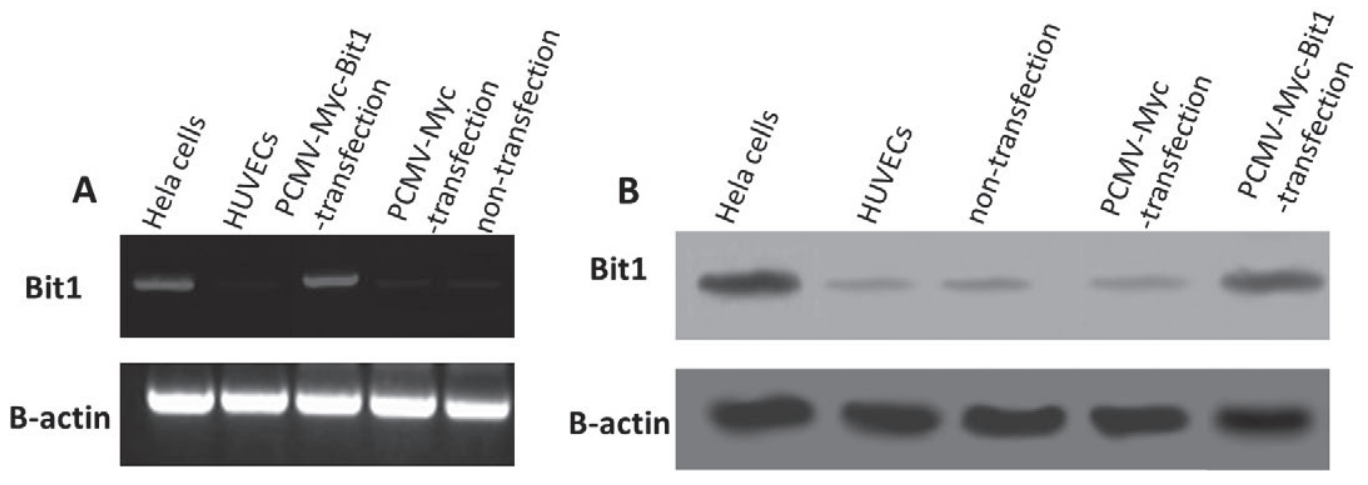

Figure 3. Bit1 mRNA (A) and protein (B) levels of cells in different groups were determined by RT-PCR and western blotting. 

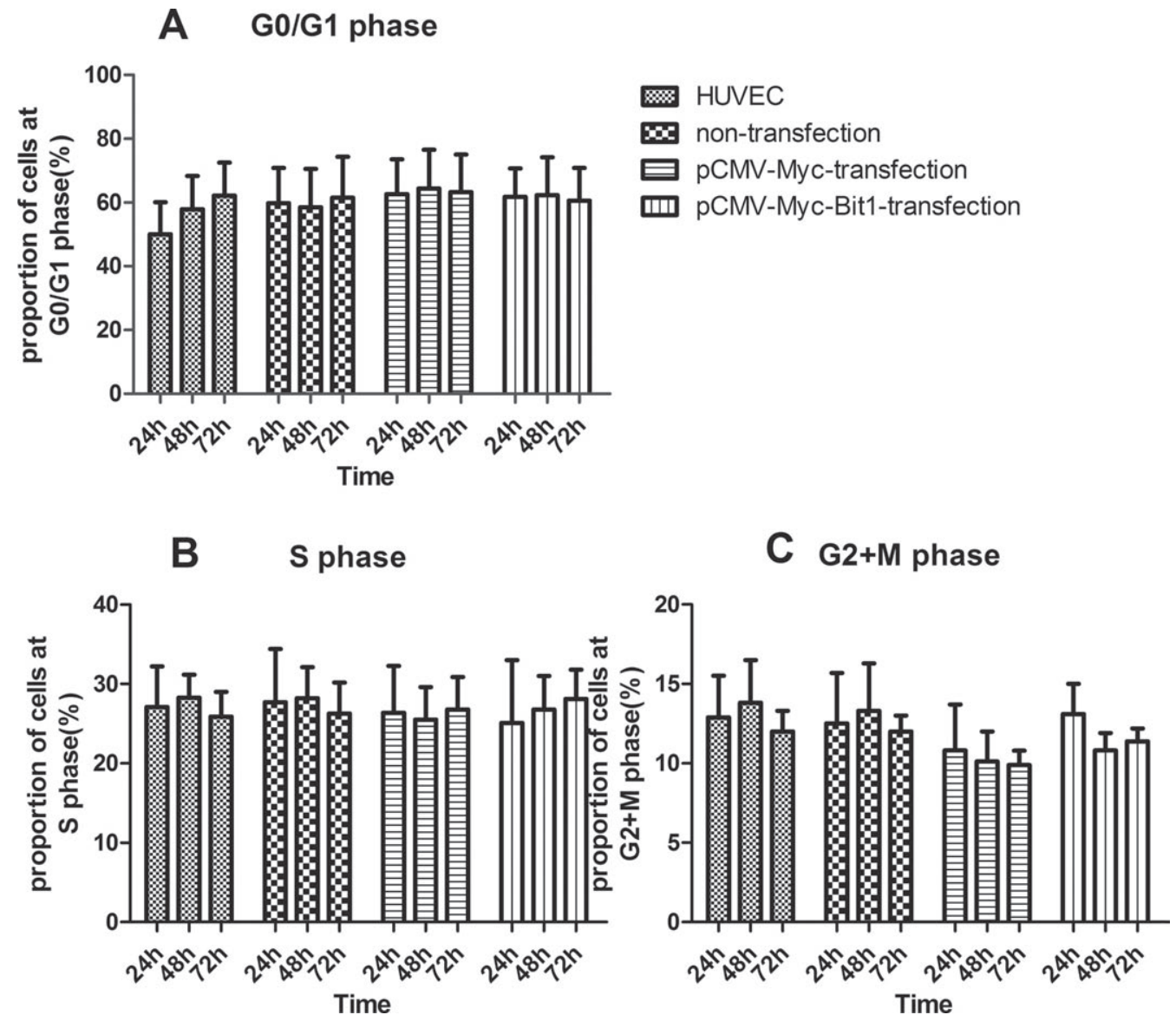

Figure 4. The proportion of cells in different phases of the cell cycle. The proportions of cells in the $G_{0} / G_{1}$ phase $(A), S$ phase $(B)$ and $G_{2}+M$ phase $(C)$ were analyzed by flow cytometry.

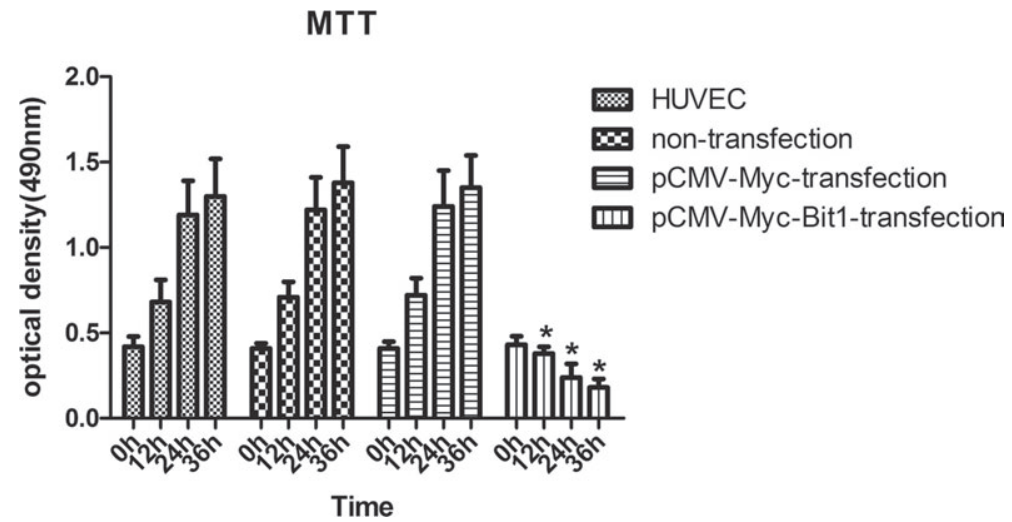

Figure 5. Cell viability was measured using $M T T$. ${ }^{\star} \mathbf{P}<0.05$, significant differences were observed between experimental group and other groups.

apoptosis[4]. So, if Bit1 is experimentally overexpressed in cytosol, it may elicit serious apoptosis. To confirm this hypothesis, we assessed cell apoptosis using different assays. Acridine orange staining showed strong and increased yellow-green fluorescence with high Bitlexpression in nucleus of most Caov-3cells (Figure 6A and B). Cells underwent specific alterations including cell shrinkage, blebbing or budding of the cell membrane, and the production of apoptotic bodies. The chromatin gathers and became pyknotic; the nuclear fragments and the chromatin were included in apoptotic 


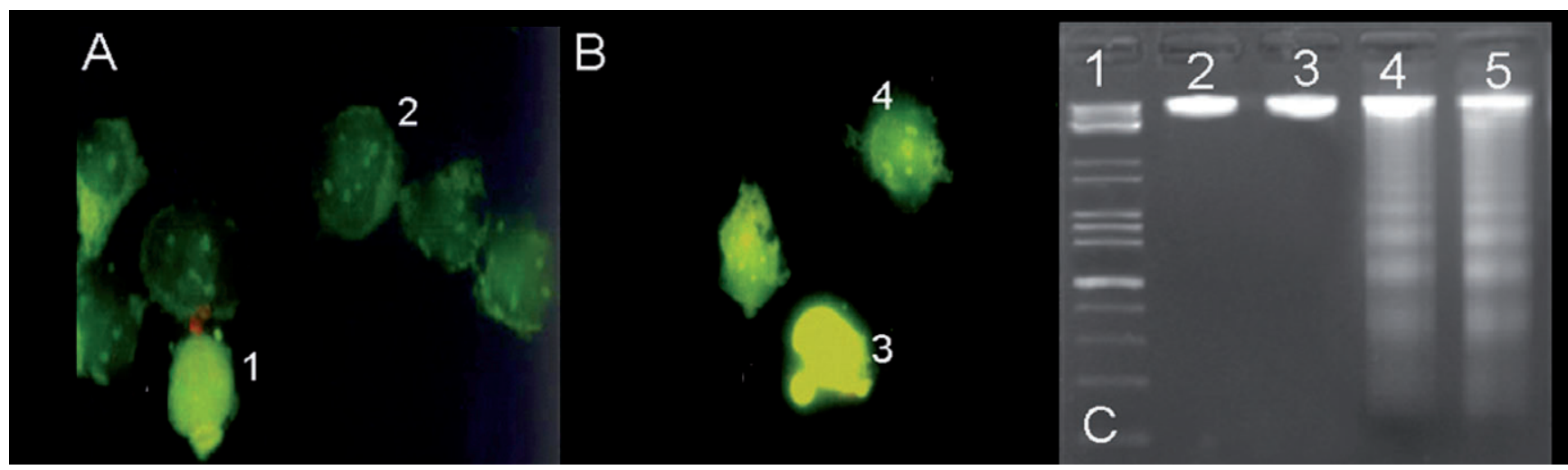

Figure 6. Cell apoptosis detected by acridine orange staining, electrophoresis and flow cytometry. A and B: Staining by acridine orange showed characteristic features of apoptosis; 1,3), apoptotic cell; 2), normal cell; 4), necrosis cell. C: apoptotic DNA ladder were detected in Caov-3 cells by agarose gel electrophoresis. 1), Marker; 2), cells without transfection; 3), pCMV-Myc transfection group; 4), positive control of U937 cells; 5), Bit1-overexpressing group.
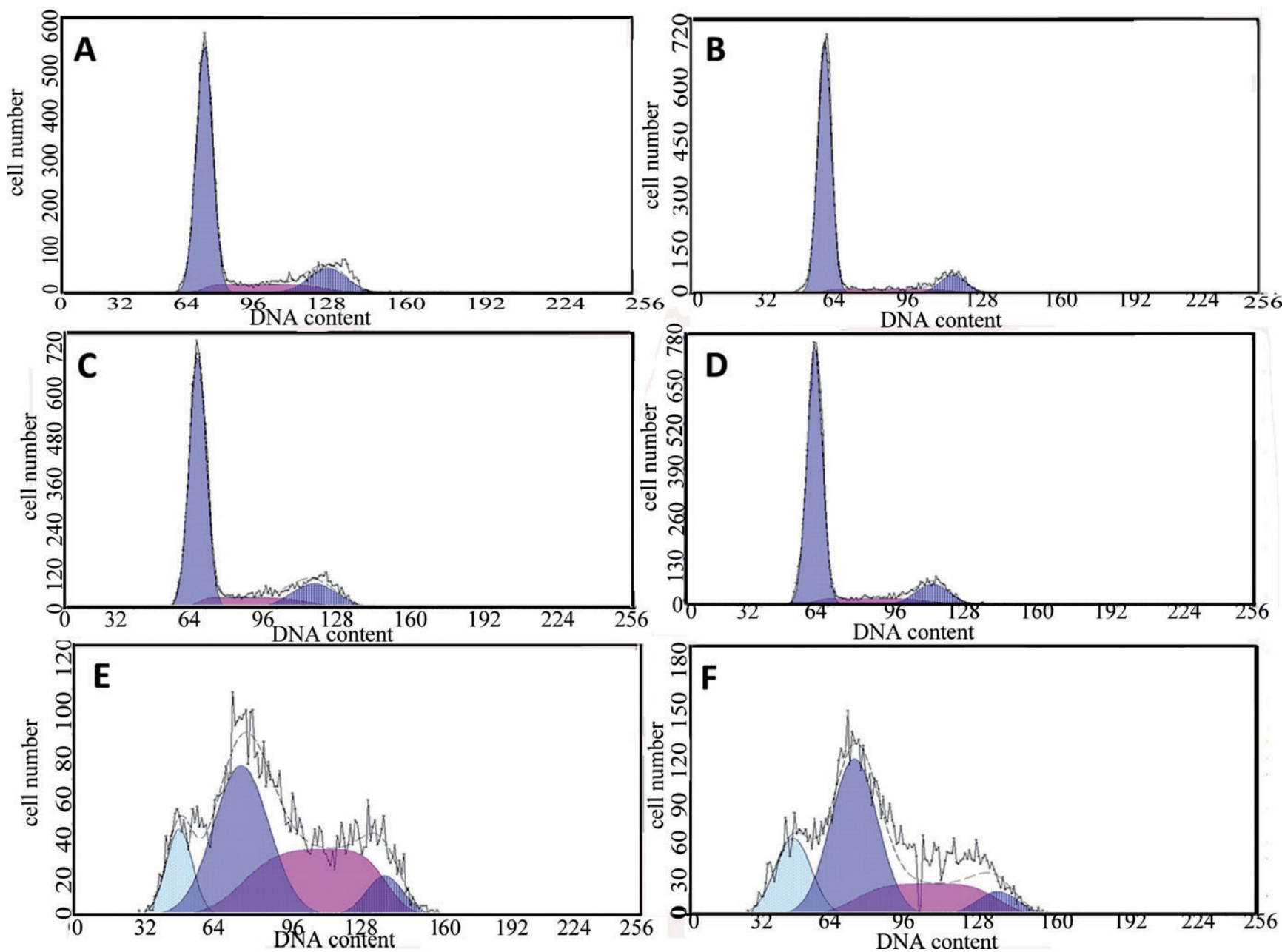

Figure 7. The cell cycle analysis. Propidium iodide (PI) staining and flow cytometric analysis were used to analysis cell apoptosis. A: Histogram of HUVECs after $72 \mathrm{~h}$ culture. B: Caov-3 cells without transfection, after 72h culture. C: Caov-3 cells transfected with pCMV-Myc, after 72h culture. D-E: Caov-3 cells trancfected with pCMV-Myc-Bit1 recombinants at $24 \mathrm{~h}(\mathrm{D}), 48 \mathrm{~h}(\mathrm{E})$ and $72 \mathrm{~h}(\mathrm{~F})$. 
bodies. Over time, the proportion of apoptotic cells increased. Agarose gel electrophoresis revealed a DNA ladder (Figure $6 \mathrm{C})$, a typical feature of apoptosis in Bit1-overexpressing cells. However at the top of the lane, non-degrading DNA was still observed after $36 \mathrm{~h}$ transfection, suggesting that only part of these cells occurred apoptosis or only part of the DNA in apoptotic cells degraded. In flow cytometry, apoptotic cells were distinguished by Sub-G1 peak on DNA histogram, this peak being a characteristic of cell apoptosis. As shown Figure 7A-C, no Sub-G1 peak emerged in HUVECs group, nontransfection group and pCMV-Myc-transfection group after $72 \mathrm{~h}$ transfection. However, after $48 \mathrm{~h}$ transfection, a Sub-G1 peak was observed $48 \mathrm{~h}$ after transfection in cells transfected with pCMV-Myc-Bit1(Figure 7E-F). In summary, these assays confirmed the pro-apoptotic action of Bitl overexpressed in the cytosol of ovarian cancer cells.

\section{Discussion}

Bit1-induced caspase-independent apoptosis has been extensively demonstrated, and increasing numbers of studies are highlighting the associated mechanisms and signaling pathways. Griffiths and colleagues demonstrated that the NF- $\kappa B$ pathway plays an important role in Bit1-mediated cell survival [14]. Hector Biliran et.al reported that protein kinase $1(\mathrm{PKD})$ is a positive regulator of Bit1 apoptotic functions [15], while according to Rania Kairouz-Wahbe, Bit1 can negatively regulate Erk activity [16]. Such investigations suggest that Bit1-induced anoikis is controlled by sophisticated regulation network, and is closely associated with other pathways and cell activities. Karmali et.al indicate that downregulation of Bit 1 confers enhanced anoikis resistance, and adhesive and migratory properties on cancer cells in vitro and specifically potentiated tumor metastasis in vivo [17]. Their findings suggest the important role of Bit1 in tumor development. However, until now, there has been a lack of data on Bit1 expression in gynaecological cancers.

Here, analyses were carried out on the Bit1 expression in relation to ovarian serous papillary adenocarcinoma. We found that the Bit 1 expression in ovarian serous papillary adenocarcinoma (100\%) was statistically higher than in normal ovarian tissues (33.3\%). Previous studies have shown that Bit1 expression was significantly higher in suspended cells than anchored cells, and that the Bit l level varies according to cell attachment [4]. In ovarian cancers cells, the cell-cell and cell-matrix attachment changed, cells lose their normal adhesion and form a new tumor microenvironment. These abnormal changes induce Bit1-release from mitochondria into cytosol and induce anoikis in tumor cell. These anoikis might be a self-protective mechanism of the body against tumor. However, a "fight or flight" mechanism against anoikis might exist in tumor cells, leading to the survival of part tumor cells and process of ovarian cancer. Analysis of the expression of Bit1 in different histologic grade of ovarian cancer suggested that there were significant differences between the
Bit 1 expression at different grade $(\mathrm{P}=0.042,0.003$; both $<0.05)$, and that Bit1 expression correlated negatively with the histologic grade. It is well known that apoptosis is the fundamental regulatory mechanism of cell numbers in ontogenesis; on the other hand, resistance to anoikis is a "fight" mechanism of tumor. Genome change in tumor cells might mean that pro-apoptosis outweigh anti-apoptosis, protecting tumor cells from apoptosis by activating the expression of a series of genes and proteins that could promote cell survival and proliferation, and by inhibiting the expression of genes that could boost cell apoptosis such as Bit1 $[18,19]$. During the development of the tumor, anti-apoptosis signals may tend to predominate, and thus the expression of pro-apoptotic molecules such as Bit1 is further constrained. These may explain the negative correlation between Bit 1 expression and histologic grade. In addition, we found that Bit1 expression was not associated with age. In this study, we also analyzed the relation between Bit 1 expression and the 5-year overall survival, and found that high histologic grade was associated with low 5-year overall survival. In summary, the expression of Bit 1 in ovarian cancer was associated with histologic grade and overall survival but not with the age. The distinct expression of Bit1 in serous papillary adenocarcinoma and normal ovarian tissues, together with the association of Bit1 expression and overall survival suggested that it may be a potential biomarker for the prediction of ovarian cancer as well as a prognostic marker for outcome.

In this study, Bit1 was experimentally overexpressed in Caov- 3 cells. The results showed that cells overexpressing Bit1 in cytosol underwent much more apoptosis than control cells and cell viability decreased, but no change in cell cycles were observed. These results confirmed the pro-apoptotic action of Bit1 and also shed lights on Bit1 as a potential gene medicine for ovarian cancer. With the recent advances in molecular virology and biotechnology, gene therapy has brought new and powerful supplements for ovarian cancer treatment. Gene therapy of ovarian cancer has been investigated intensively $[20,21]$. The direct introduction of Bit1 into ovarian cancer to induce cell apoptosis may provide a new approach to ovarian cancer treatment. As more and more tumor-specific molecules or antigens being found, the tumor-targeted therapy has been receiving increasing attentions. It is likely that gene therapy will be incorporated into tumor-targeted strategies in an attempt to enhance tumor cell death, generate potent anti-tumor immune responses, and suppress angiogenesis.

In summary, our study showed a close relationship between Bit1 and ovarian serous papillary adenocarcinoma for the first time. As a pathologically relevance factor of serous papillary adenocarcinoma, Bit 1 will be a predictor of prognosis and outcome as well as a potential gene medicine for serous papillary adenocarcinoma. Further functional analyses of Bit1 in these and other studies will help to usher us into an age of personalized therapy for serous papillary adenocarcinoma. 


\section{Reference}

[1] HEINTZ A, ODICINO F, MAISONNEUVE P, BELLER U, BENEDET J, et al.: Carcinoma of the ovary. International Journal of Gynaecology and Obstetrics 2003; 83: 135-166. http://dx.doi.org/10.1016/S0020-7292(03)90118-4

[2] MOSS C, KAYE SB: Ovarian cancer:: Progress and continuing controversies in management. European Journal of Cancer 2002; 38: 1701-1707. http://dx.doi.org/10.1016/S09598049(02)00161-2

[3] SIMPSON CD, ANYIWE K, SCHIMMER AD: Anoikis resistance and tumor metastasis. Cancer letters 2008; 272: 177-185. http://dx.doi.org/10.1016/j.canlet.2008.05.029

[4] JAN Y, MATTER M, PAI J, CHEN YL, PILCH J, et al.: A mitochondrial protein, bit1, mediates apoptosis regulated by integrins and groucho/tle corepressors. Cell 2004; 116: 751-762. http://dx.doi.org/10.1016/S0092-8674(04)00204-1

[5] LAI CH, CHOU CY, CH'ANG LY, LIU CS, LIN W: Identification of novel human genes evolutionarily conserved in caenorhabditis elegans by comparative proteomics. Genome research 2000; 10: 703-713. http://dx.doi.org/10.1101/ gr.10.5.703

[6] CHEN G, COUREY AJ: Groucho/tle family proteins and transcriptional repression. Gene 2000; 249: 1-16. http://dx.doi. org/10.1016/S0378-1119(00)00161-X

[7] SHEPHERD JH: Revised figo staging for gynaecological cancer. BJOG: An International Journal of Obstetrics \& Gynaecology 1989; 96: 889-892. http://dx.doi.org/10.1111/ j.1471-0528.1989.tb03341.X

[8] XIE X, WANG CY, CAO YX, WANG W, ZHUANG R, et al.: Expression pattern of epithelial cell adhesion molecule on normal and malignant colon tissues. World J Gastroenterol 2005; 11: 344-347.

[9] AUST DE, TERDIMAN JP, WILLENBUCHER RF, CHANG CG, MOLINARO-CLARK MS, et al.: The apc/ $\beta$-atenin pathway in ulcerative colitis-related colorectal carcinomas. Cancer 2002; 94: 1421-1427. http://dx.doi.org/10.1002/ cncr.10334

[10] HUA W, CHEN B, ZHANG W, MIAO S, ZHANG H, et al.: Monoclonal antibodies against human bit 1, an apoptosis-associated mitochondrial protein. Hybridoma 2009; 28: 167-171. http://dx.doi.org/10.1089/hyb.2008.0095
[11] TADA H, SHIHO O, KUROSHIMA K, KOYAMA M, TSUKAMOTO K: An improved colorimetric assay for interleukin 2. Journal of immunological methods 1986; 93: 157-165. http://dx.doi.org/10.1016/0022-1759(86)90183-3

[12] DARZYNKIEWICZ Z, BEDNER E, SMOLEWSKI P: Flow cytometry in analysis of cell cycle and apoptosis, Elsevier, 2001, 38, pp 179-193.

[13] NUNEZ R: DNA measurement and cell cycle analysis by flow cytometry. Current issues in molecular biology 2001; 3: 67-70.

[14] GRIFFITHS GS, GRUNDL M, LEYCHENKO A, REITER S, YOUNG-ROBBINS SS, et al.: Bit-1 mediates integrin-dependent cell survival through activation of the $\mathrm{nf}-\kappa \mathrm{b}$ pathway. Journal of Biological Chemistry 2011; 286: 14713-14723. http://dx.doi.org/10.1074/jbc.M111.228387

[15] BILIRAN H, JAN Y, CHEN R, PASQUALE EB, RUOSLAHTI E: Protein kinase $\mathrm{d}$ is a positive regulator of bit1 apoptotic function. Journal of Biological Chemistry 2008; 283: 2802928037. http://dx.doi.org/10.1074/jbc.M803139200

[16] KAIROUZ-WAHBE R, BILIRAN H, LUO X, KHOR IW, WANKELL M, et al.: Anoikis effector bit1 negatively regulates erk activity. Proceedings of the National Academy of Sciences 2008; 105: 1528-1532. http://dx.doi.org/10.1073/ pnas.0711357105

[17] KARMALI PP, BRUNQUELL C, TRAM H, IRELAND SK, RUOSLAHTI E, et al.: Metastasis of tumor cells is enhanced by downregulation of bit1. PloS one; 6: e23840. http://dx.doi. org/10.1371/journal.pone.0023840

[18] MANDAI M, MATSUMURA N, BABA T, YAMAGUCHI K, HAMANISHI J, et al.: Ovarian clear cell carcinoma as a stressresponsive cancer: Influence of the microenvironment on the carcinogenesis and cancer phenotype. Cancer letters 2011; 310:129-133. http://dx.doi.org/10.1016/j.canlet.2011.06.039

[19] VAUX DL: Apoptogenic factors released from mitochondria. Biochimica et Biophysica Acta (BBA)-Molecular Cell Research 2011; 1813: 546-550.

[20] COUKOS G, RUBIN S: Gene therapy for ovarian cancer. Oncology 2001; 15: 1197-1205.

[21] CANNON MJ, SANTIN AD, O'BRIEN TJ: Immunological treatment of ovarian cancer. Current Opinion in Obstetrics and Gynecology 2004; 16: 87. http://dx.doi.org/10.1097/ 00001703-200402000-00015 